\title{
A New Shaped Waveform Design Scheme for Radar Embedded Communication
}

\author{
Shan $\mathrm{He}^{1}$, Baoguo $\mathrm{Li}^{1}{ }^{1}$,Jing $\mathrm{Lei}^{1}$,Hua Yan ${ }^{1}$ \\ ${ }^{1}$ School of Electronic Science,National University of Defense Technology, Changsha, Hunan Province, \\ People's Republic of China
}

\begin{abstract}
In order to improve the covertness of radar embedded communication based on the eigenvalue decomposition radar embedded communication waveform design scheme, a new waveform design scheme that combines the idea of shaped design is constructed. In this paper, the background and concept of radar embedded communication are introduced in detail. Three existing waveform design schemes are analyzed. Using de-correlation filter receivers, a weighted waveform design scheme for shaped non-dominant subspace feature vectors is constructed and analyzed. The communication covertness and reliability is illustrated. Both the theoretical analysis and the simulation show that this scheme improves the covertness of communication.
\end{abstract}

\section{Introduction}

Most of the existing stealth technologies use signals that have already existed in the environment. In addition to noise, radar signals can also be used as a carrier for concealing communication signals. Radar signal is an ideal tool for realizing stealth communication. Radar Embedded Communication (REC) is a stealth communication technology that uses radar backscatter signals to hide communication signals. It has low probability of detection (LPD) and low probability of interception (LPI). The basic principle of REC is described as follows: The friend illuminator launches the radar detection waveform. After receiving the radar signal from the tag, the tag sends out the REC signal. At the same time, the radar detection waveform touches obstacles such as trees, rivers, and buildings to generate radar backscatter. During the process, useful information is embedded into the normal radar backscatter as a mask. The intended receiver can extract the communication while intercept receiver fails, as shown in Fig. 1.

The concept of the REC originated from the Shannon D. Blunt team at the University of Kansas. The team used a so-called inter-pulse radar embedded communication method to carry information amongst pulses at first.It mainly utilised radar illumination consisting of numerous of pulses as is encountered in synthetic aperture radar

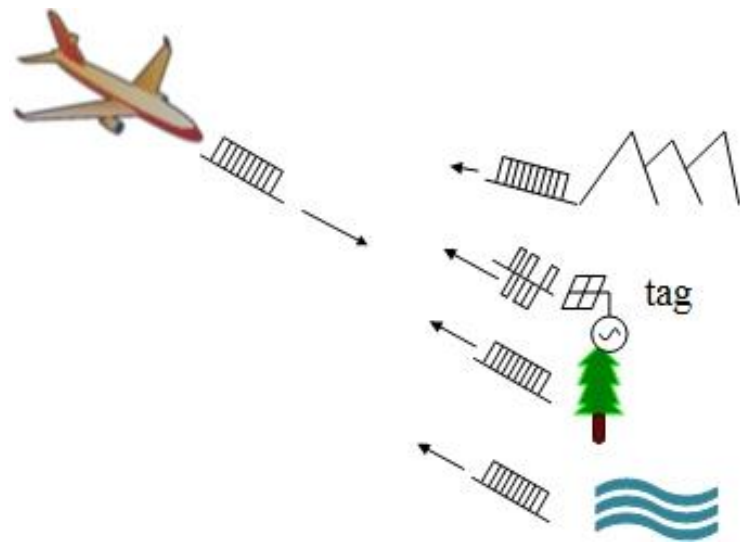

Fig1. Paradigm of REC

Applications, but the intra-pulse radar embedded communication rate is limited. Subsequently, the team adopted a radar embedded communication method based on the LFM pulse and proposed three waveform design schemes based on eigenvalue decomposition. Padmaja Yatham later conducted an analysis and discussion of these three methods [1]. Justin G. Metcalf proposed a detection strategy based on intra-pulse radar embedded communication and proposed a covert evaluation metric [2]. At the same time, other waveform design schemes were proposed. Li Baoguo from the National University of Defense Technology proposed a radar embedded communication waveform design method based on a direct-spread-spectrum sequence. It used direct-spreadspectrum sequence to enhance communication reliability [3]. Shannon D. Blunt team used the WF waveform 
proposed by the water filling principle [4]. D. Ciuonzo used the Pareto theory to discuss the mutually contradictory indicators of reliable communication and covertness of radar embedded communication [5], and continued to use the multi-objective optimization theory to further the two metrics [6]. The paper proposed the use of sparse frequency methods for waveform design in [7]. The paper combined CPM and PCFM to embed communication signals into radar signals in [8].

\section{Waveform design scheme based on eigenvalue decomposition}

When analyzing the radar signal, the tag will oversample the received radar detection signal, assuming that the Nyquist sampled radar waveform has a time-bandwidth product $N$ and is oversampled at $M_{c}$ Nyquist rate times. The sample radar waveform is represented as a vector of lengths $N M_{c}$ :

$$
s=\left[s_{1}, s_{2}, s_{3} \cdots s_{N M_{c}}\right]
$$

The discrete clutter distribution is

$$
s_{n}=\exp \left(\frac{j \pi n^{2}}{N M_{c}}\right)
$$

When considering the convolution of $s$, it is noticed that there is a $2 N M_{c}-1$ possible shift. Therefore, the Toeplitz matrix $S$ is defined as:

$$
\mathbf{S}=\left[\begin{array}{cccccc}
s_{N M_{\mathrm{c}}} & s_{N M_{\mathrm{c}}-1} & \cdots & s_{1} & \cdots & 0 \\
0 & s_{N M_{\mathrm{c}}} & \cdots & s_{2} & \cdots & 0 \\
\vdots & \vdots & \ddots & \vdots & \cdots & \vdots \\
0 & 0 & \cdots & s_{N M_{\mathrm{c}}} & \cdots & s_{1}
\end{array}\right]
$$

One of the desirable properties of the waveforms is to make them naturally dependent or similar to backscatter, so it can be concealed using radar backscattering. Therefore, the strategy of the waveform design is to use the eigenspace of the radar reflection to maintain the correlation with the clutter. The eigenvalue decomposition of $\mathrm{S}$ is performed before the waveform is constructed:

$$
S S^{H}=V \Lambda V^{H}=\left[V_{N D} \mid V_{D}\right]\left[\begin{array}{cc}
\Lambda_{N D} & 0 \\
0 & \Lambda_{D}
\end{array}\right]\left[\frac{V_{N D}}{V_{D}}\right]
$$

$V_{N D}$ is a $N M_{c} * L$ matrix of $L$ non-dominant eigenvectors, $V_{D}$ is of dimension $N M_{c} *\left(N M_{c}-L\right)$ and consists of dominant eigenvectors, $\Lambda_{N D}$ is a $L * L$ diagonal matrix of the $L$ non-dominant eigenvalues and $\Lambda_{D}$ is a $\left(N M_{c}-L\right) *\left(N M_{c}-L\right)$ diagonal matrix of dominant eigenvalues. For spectrum, the eigenvector with the larger eigenvalue has the energy component of the larger part of the radar signal, which means that the eigenvector in $V_{D}$ represents the frequency component in the radar passband and the eigenvector in $V_{N D}$ represents the radar in the stopband of frequency component.

The current three radar-embedded communication waveform design scheme use eigenvalue decomposition as means: non-dominant eigenvectors as communication
waveforms(EAW), dominant projection waveforms(DP), and weighted-combing waveforms(WC) will be discussed in the next chapter, the analysis of the first two schemes is as follows.

\subsection{Eigenvectors \\ as \\ communication waveforms (EAW)}

In order to facilitate the cooperative receiver to detect the communication signal sent by the tag, the waveforms should be orthogonal to each other, and the eigenvectors are orthogonal to each other. Therefore, eigenvectors are directly used as communication waveforms. The waveforms designed by this method are also orthogonal to each other. Therefore, the non-dominant eigenvectors in $V_{N D}$ are directly used as communication waveforms:

$c_{k}=V_{k} \quad k=1,2 \cdots K$

In order to minimize radar interference, the eigenvectors corresponding to the first $\mathrm{K}$ smallest eigenvalues are selected. These eigenvectors originate from a non-dominant subspace, which makes the difference between the communication waveforms and the radar waveforms maximized. This ensures that the cooperative receiver can quickly extract the communication waveform. This waveform scheme has high communication reliability. In turn, it will also make it easier for the interceptor to detect and identify the communication waveforms. Fig. 2 shows the EAW waveform and radar waveform spectrum. It can be seen that the EAW waveform has a sharp peak in the frequency spectrum, making the communication waveform easy to intercept.

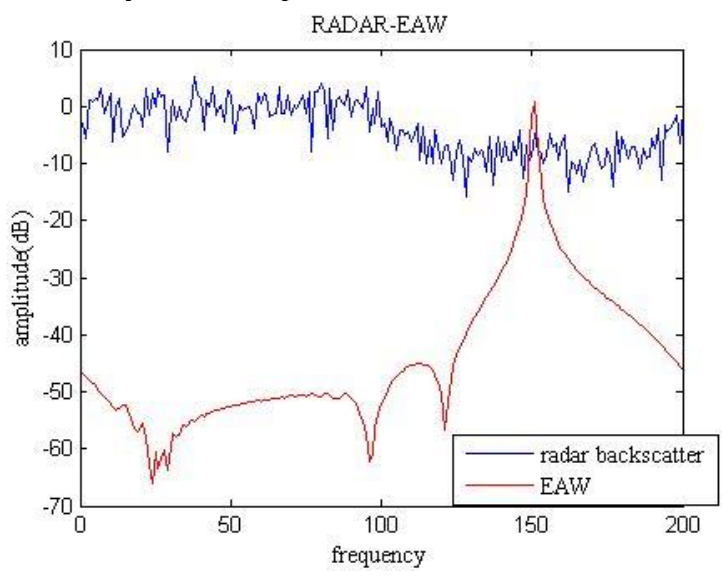

Fig2. Spectrum of radar backscatter and EAW waveform

\subsection{Dominant projection (DP)}

The previous communication waveform design scheme EAW operates on non-dominant eigenvectors. Now orthogonal projection is used to process the dominant subspace to generate a projection matrix $P_{1}=I-V_{D} V_{D}^{H}$, which we also call it hidden matrix $P$. Then the projection matrix $P_{1}$ is multiplied by a random column vector $d_{1}$ known by both the tag and the cooperating 
receiver to generate the communication waveform $c_{1}$ :

$$
c_{1}=P_{1} d_{1}
$$

Build a the new matrix $S_{P 1}=\left[S \mid c_{1}\right]$. Then perform eigenvalue decomposition on the new topliz matrix $S_{P 1}$

$$
S_{P 1} S_{P 1}^{H}=V_{P 1} \Lambda_{P 1} V_{P 1}^{H}
$$

Get a new projection matrix $P_{2}$ again, in order to remove the effect of the previous waveform:

$$
P_{2}=I-V_{P 1, D} V_{P 1, D}^{H}
$$

Then build the next radar embedded communication waveform $c_{2}$ :

$$
c_{2}=P_{2} d_{2}
$$

The above process is cycled $K$ times to obtain radar embedded communication symbols and $K$ DP waveforms are obtained. The DP waveform spectrum is showed in Fig.3.

According to the spectrum diagram, after introducing the concept of the projection matrix, the communication waveform is closer to the noise in the stopband, and the power of the radar embedded waveform received is lower than the power of the radar backscatter, so the backscatter can be used to ensure greater covertness. RADAR-DP

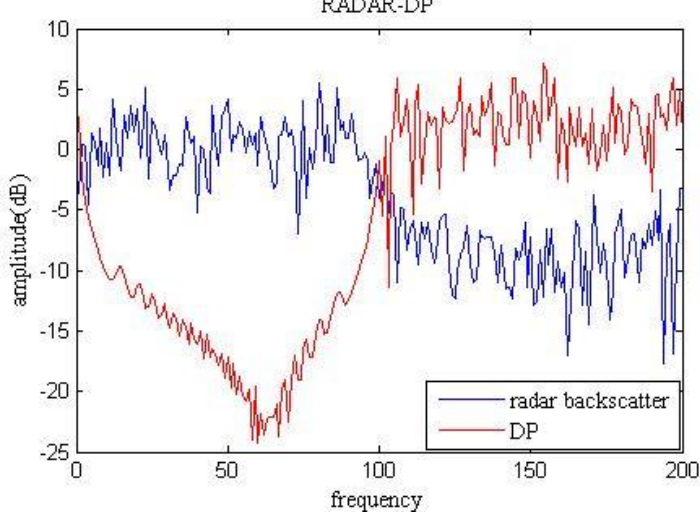

Fig3. Spectrum of radar backscatter and DP waveform

\section{3 weighted-combing wavEform}

\subsection{WC waveform design.}

As discussed in the previous chapter, EAW uses the eigenvectors in non-dominant subspace directly as the communication waveform, and there will be a obvious peak on the spectrum diagram, leading to poor covertness. In order to enhance the covertness of communication waveforms, WC waveform design adopts all nondominant subspace eigenvectors to design waveforms.

$$
V_{N D}=\left(V_{1}, V_{2} \cdots V_{L}\right)
$$

$L$ represents the size of the non-dominant subspace. Then $K$ communication waveforms can be generated by the following combinations:

$$
c_{k}=V_{N D} \cdot b_{k} \quad k=1,2 \cdots K
$$

$b_{k}$ is a random column vector of length $L$. The $b_{k}$ message is only known to the cooperative receiver.
Adopting all the non-dominant subspace eigenvectors will make the radar waveform and communication waveform more similar, and the covertness is greater compared with EAW, which will make the interceptor more difficult to detect and recognize. Fig.4 shows the WC waveform and radar waveform spectrum.

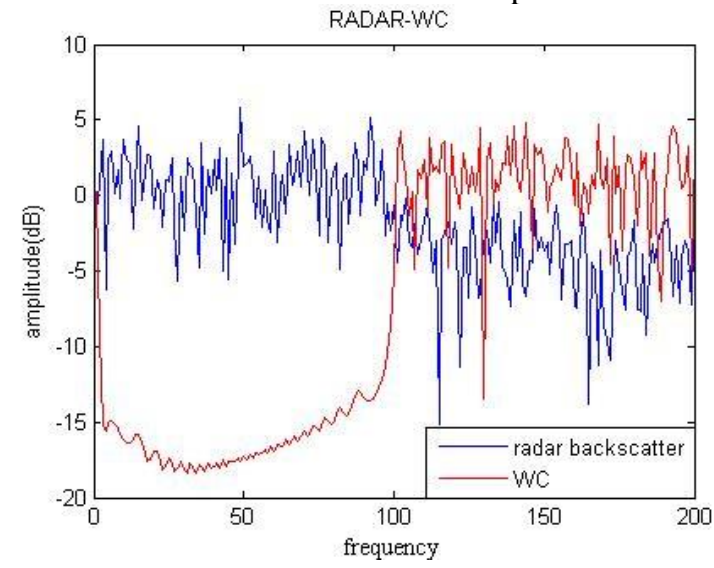

Fig4. Spectrum of radar backscatter and WC waveform

\subsection{Shaped weighted-combing (SWC)}

The above waveform design scheme considers only the eigenvectors but does not consider the eigenvalues. The shaped waveform design scheme starts from the eigenvalues. The eigenvalues are based on the sum of the noise and clutter of each eigenchannel, and the spectrum is covered by the communication symbols. At the same time, the spectral components in the stopband of the radar signal can be imitated more closely. Therefore, the scheme of shaped waveforms is adopted. The eigenvalue matrix $\Lambda_{N D}$ is integrated into the waveform design scheme:

$$
c_{k}=V_{N D} \cdot\left(\Lambda_{N D}\right)^{m} \cdot b_{k} \quad k=1,2 \cdots K
$$

$\Lambda_{N D}$ cannot be used directly in waveform design. We consider $\Lambda_{N D}$ to give different powers for discussion because $\Lambda_{N D}$ is a non-dominant subspace eigenvalue matrix .It is a part of the $\Lambda$ eigenvalue matrix, and the $\Lambda$ eigenvalue plot is shown in Fig.5.

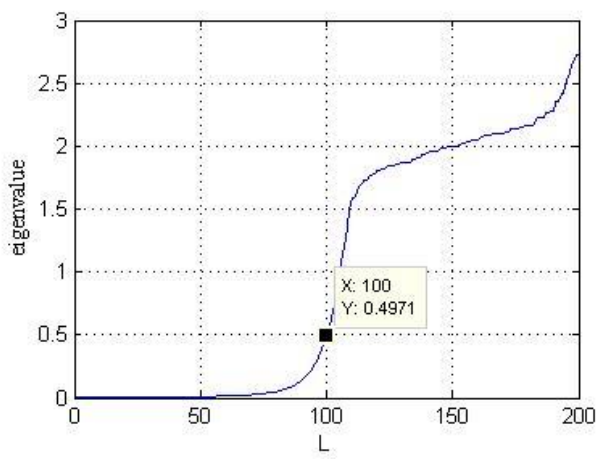

Fig5. Eigenvalue plot of intra-plused radar

As can be seen from the figure, $\Lambda_{N D}$ is a typical diagonal matrix whose diagonal elements increase from 0 
to 0.5 in order, giving $\Lambda_{N D}$ different power, which can change the spectrum of the communication waveform. The communication waveform can imitate the spectral component of the stopband as much as possible on the basis of satisfying reliable communication. With the gradual increase of $m$, the elements of the $\Lambda_{N D}$ diagonal matrix begin to decrease. When $m=0, \Lambda_{N D}$ is the identity matrix, and the SWC is the same as the WC. When $m \geq 0.5$, the spectrum of the communication waveform is gradually submerged under the radar waveform, resulting in the communication waveform being difficult to be reliably received by the cooperative receiver, and the communication reliability is poor, as shown in Fig.6 and Fig. 7. When $m=0.25$ the communication waveform is not overwhelmed by the radar waveform and the performance is ideal, as shown in Figure 8.

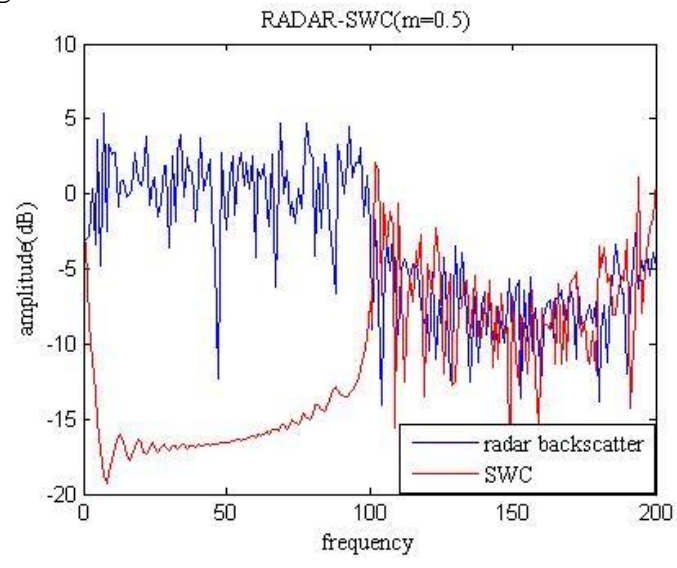

Fig6. Waveform of $\mathrm{SWC}(\mathrm{m}=0.5)$

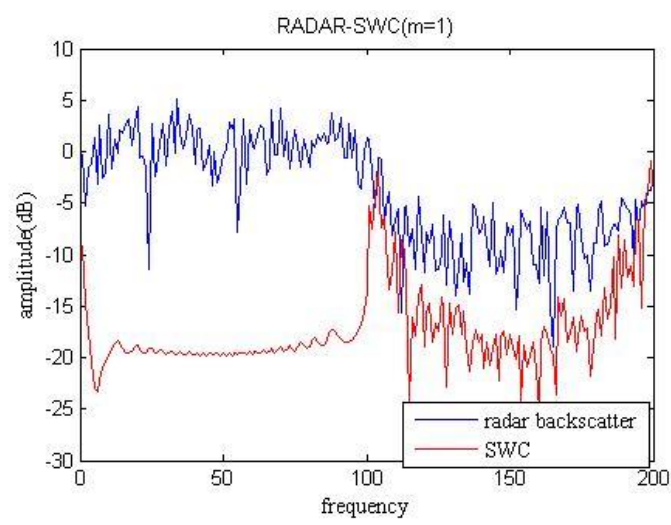

Fig7. Waveform of $\operatorname{SWC}(\mathrm{m}=1)$

When $m=0.25$, the SWC waveform is;

$$
c_{k}=V_{N D} \cdot\left(\Lambda_{N D}\right)^{0.25} \cdot b_{k}
$$

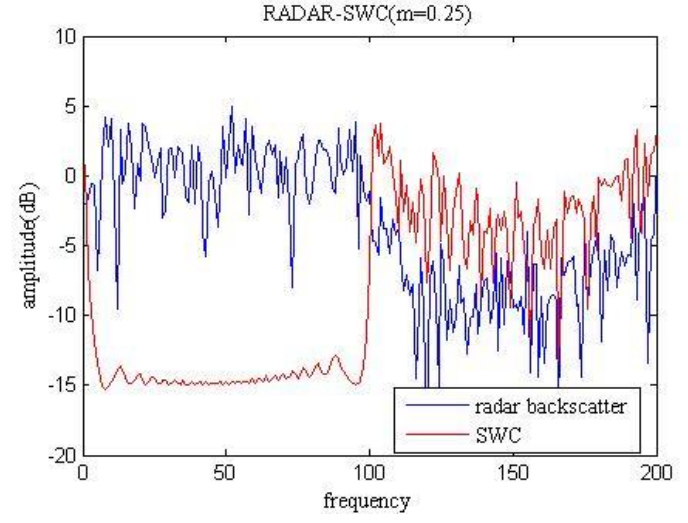

Fig8. Spectrum of radar backscatter and SWC waveform

For the shaped matrix $\left(\Lambda_{N D}\right)^{m}$, in the case of $m=0.25$, diagonal elements are arranged from small to large in order of 0.26 to 0.83 . As shown in Fig.9, the shaped matrix reduces the eigenvector matrix in proportion. Each eigenvector $\alpha$ in $V_{N D}$ is proportionally reduced according to the diagonal elements of the shaped matrix $\left(\Lambda_{N D}\right)^{m}$, so that the waveform is closer to the frequency components in the radar stopband, and thus the covertness of the radar embedded communication waveform can be significantly improved.

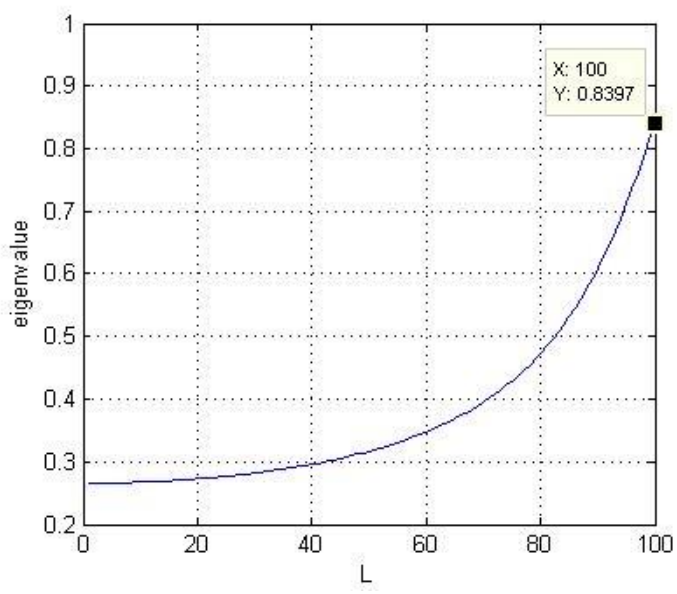

Fig9. Eigenvalue plot of shaped matrix $\left(\Lambda_{N D}\right)^{m}$

\section{Simulation Results}

In the waveform design scheme of radar embedded communication, the reliability and covertness of communication are mutually antagonistic. The higher the communication reliability is, the more easily it will be received by the cooperative receiver, and it will be more easily intercepted by the interceptor, so the covertness is poor. This chapter evaluates radar embedded communication waveform design schemes in terms of communication reliability and communication covertness. 


\subsection{Communication Reliability}

Radar embedded communication waveforms only involve the decision process of the communication waveform. They do not involve waveform symbol mapping, use the existing signal-to-noise ratio SIR (communication signal to ambient noise ratio) and signalto-noise ratio SCR (communication signal power and radar backscatter power ratio), we use the receiver's symbol error rate(SER) to measure the reliability of communications.

In [2], it has been proved that the decorrelation filter has better reception performance than the matched filter. Therefore, in the measurement of communication reliability, this paper uses a decorrelation filter as an example for analysis.

Build the filter as follows:

$$
w_{k}=\left(S S^{H}+\delta_{M A X} I\right)^{-1} c_{k}
$$

In the above formula, $\delta_{M A X}$ is the maximum nondominant eigenvalue, and $I$ is the identity matrix. The decision uses the filter $w_{k}$ in place of $c_{k}$, and performs correlation calculation processing with the filter and the received signal to obtain the most relevant waveform as the determination waveform, then the communication symbol is obtained.

$$
\hat{k}=\arg \left\{\max _{k}\left(\left|w_{k}^{H} \bullet r\right|\right)\right\} \quad k=1,2 \cdots K
$$

For the decorrelation filter, the symbol error rates for the above four communication waveforms are shown in the figure. The simulation uses the main space size $L=100$, the sampling points $N=100$, the oversampling ratio $M_{c}=2$, and the simulation uses $10^{6}$ in the Monte Carlo method, the channel used is Gaussian white noise channel. The simulation results are shown in Fig.10.

According to the simulation image, the SER performance EAW of the four radar embedded waveform design schemes is greater than WC, DP and SWC.

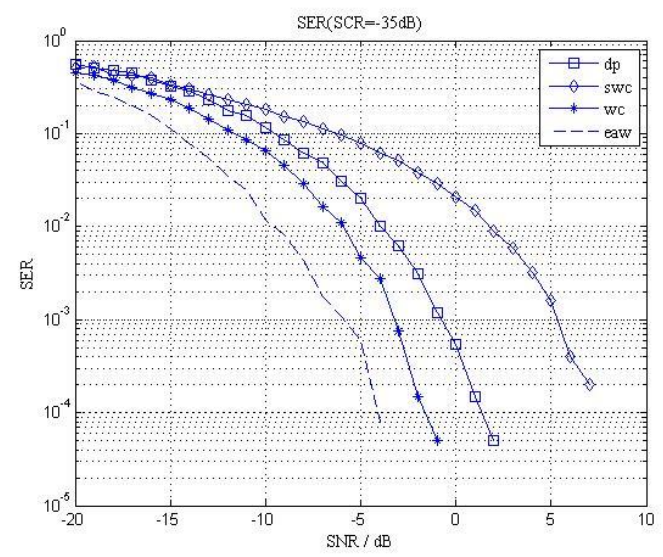

Fig.10 SER for four REC waveforms $(\mathrm{SCR}=-35 \mathrm{~dB})$

\subsection{Communication covertness}

In the previous section, the SER simulation results of four types of waveforms are discussed, which can quantitatively reflect the communication reliability of these four types of waveforms. In this section, we will discuss the communication covertness of each waveform design scheme, the measure of the low probability of interception (LPI). Because intra-pulse radar embedded communications operate by inserting a communication waveform within or around the spectrum occupied by radar backscatter.So the classical concept of intercept probability based on spectral energy content measurements no longer applies.

Reference [2] proposes to combine the prediction waveform $z$ with the communication waveform $c_{k}$ and use the correlation coefficient corr to quantify communication covertness. Although the evaluation metric does not directly determine the probability of interception, it can be used to infer whether the interceptor can intercept communication waveforms. The higher the correlation coefficient, the more likely it is to intercept radar embedded waveforms, and conversely the smaller the probability of interception.

$\eta_{k, j}=\frac{\left|z_{j} c_{k}\right|}{\sqrt{z_{j}^{H} z_{j}} \sqrt{c_{k}^{H} c_{k}}}$

The receiver does not know the size of the nondominant subspace $L$ that constitutes the hidden matrix during the waveform design process. First, the size of the non-dominant space need to be predicted, and then the hidden matrix $P_{j}$ can be constructed.

$$
P_{\mathrm{j}}=\tilde{V}_{N D, j} \tilde{V}_{N D, j}^{H}
$$

After the interceptor receives the signal $r$ through the hidden matrix, the predicted waveform $z$ is obtained.

$$
\tilde{z}_{j}=P_{j} \bullet r
$$

After the prediction waveform $\mathrm{z}$ is obtained, the number of simulation sampling points is $N=100$, $M_{c}=2$, the non-dominant subspace $L$ space size is $L=100, \mathrm{SCR}=-35 \mathrm{~dB}, \mathrm{SNR}=0 \mathrm{~dB}$, and the correlation coefficient graph is obtained after matlab simulation. The simulation results are shown in Fig.11.

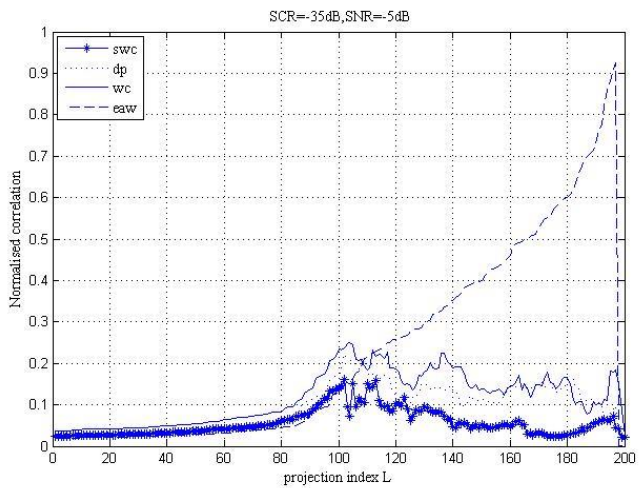

Fig.11 Normalised correlation for four REC waveforms $(\mathrm{SCR}=-$ $35 \mathrm{~dB}, \mathrm{SNR}=-5 \mathrm{~dB}$ 
Through the simulation graph, we can see that the EAW directly uses the non-dominant subpace eigenvectors as the communication waveform to obtain a correlation coefficient close to 1 , indicating that the EAW does not have covertness, which is consistent with the theoretical analysis results. The correlation coefficients of WC, DP, and SWC are all relatively low, and the highest point appears near $\mathrm{L}=100$. The correlation coefficient curve of SWC is the lowest and is always lower than 0.2 , so it can be concluded that the LPI performance of SWC waveform is better than that of WC and DP.

\section{Conclusions}

Both theoretical analysis and experimental simulations show that using the design scheme of shaping and introducing the non-dominant subspaces eigenvalues diagonal matrix into the waveform design scheme can significantly improve the covertness of radar embedded communication waveforms. A new waveform design scheme called shaped weighted combing (SWC)is constructed. This waveform design scheme has greatly improved covertness compared to other three waveform design methods, and the waveform design scheme is simple and easy to implement, and the price paid is the reduction of communication reliability, if the communication reliability is appropriately improved. We can consider the direct-spread-spectrum method proposed in [3] to improve communication reliability.

\section{References}

1. S. D. Blunt,Yatham P., 2007. Waveform Design for Radar-embedded Communications[C]. International Waveform Diversity and Design Conference, 214218.

2. Metcalf J,Blunt S D,Perrins E. 2011. Detector Design and Intercept Metrics for Intra-Embedded Communications[C].The $2011 \quad$ Military Communication Conference, 188-192

3. Baoguo Li,Jing Lei, Wei Cao, 2016. Waveform Design for Radar-embedded Communications Exploiting Spread Spectrum Technology[J]. IET Communications, 1631-1639.

4. Justin G,Metcalf,Cenk Sahin,et al, 2015. Analysis of Symbol-Design Strategies for Intra Pulse Radarembedded Communications[J].Aerospace and Electronic Systems, 2914-2931.

5. Ciuonzo D,De Maio A,Foglia G,et al, 2015. Paretotheory for Enabling Covert Intra Pulse Radarembedded Communications[C]. Proceedings of IEEE International Radar Conference, , 292-297.

6. Ciuonzo D,De Maio A,Foglia G,et al, 2015. Intrapulse Radar-embedded Communications Via Multiobjective Optimization [C]. IEEE Transactions on Aerospace and Electronic Systems, 2960-2967.
7. Chaoyun Mai,Jinping Sun,Rui Zhou, 2016. Sparse Frequency Waveform Design for Radar-Embedded Communication $[\mathrm{J}]$. Hindawi Publishing Corporation, 72700301.

8. C.Sahin,J.Jakabosky,P.M.McCormick,J.G. Metcalf,S.D. Blunt, 2017. A Novel Approach for Embedding Communication Symbols into Physics Radar Waveforms [C]. IEEE Radar Conference, 214-221. 